\title{
Extensão universitária na ótica de acadêmicos: o agente fomentador das Diretrizes Curriculares Nacionais
}

Suzely Adas Saliba Moimaz*; Danielle Bordin**; Adrielle Mendes de Paula Gomes**; Cristina Berger Fadel***; Cléa Adas Saliba Garbin*; Nemre Adas Saliba*

* Professora Titular do Departamento de Odontologia Infantil e Social da Faculdade de Odontologia de Araçatuba da Universidade Estadual Paulista

** Aluna de Doutorado do Programa de Odontologia Preventiva e Social da Faculdade de Odontologia de Araçatuba da Universidade Estadual Paulista

*** Professora Adjunta do Departamento de Odontologia da Universidade Estadual de Ponta Grossa

\section{RESUMO}

Objetivou-se analisar a percepção de acadêmicos de Odontologia acerca de um programa de extensão, sob a ótica da aproximação com as Diretrizes Curriculares Nacionais (DCN) do referido curso. Trata-se de um estudo qualitativo, desenvolvido com acadêmicos $(n=5)$ participantes de um programa de atenção à gestante. Os dados foram obtidos por meio de grupo focal e analisados pela Técnica do Discurso do Sujeito Coletivo. Foi possível observar que o impacto exercido pela vivência acadêmica em atividades extensionistas aprimora o desenvolvimento científico, técnico e de relações humanas. No campo do desenvolvimento científico, o ganho foi no recebimento de estímulos para desenvolver suas aptidões para buscar novos conhecimentos e fixar o conteúdo teórico na vivência prática. Quanto ao desenvolvimento técnico, houve aprimoramento do conhecimento técnicocientífico e prático para a atenção humanizada às gestantes, além do desenvolvimento de habilidades motoras, cognitivas (para o entendimento do funcionamento do sistema público de saúde) e de comunicação e expressão do acadêmico. No campo do desenvolvimento das relações humanas, o profícuo relacionamento estabelecido entre acadêmico, universidade e sociedade permitiu incrementar as habilidades de trabalhar em equipe e o senso de cidadania. Conclui-se que um programa de extensão se configura como excelente oportunidade de formação profissional, mais contextualizada com as habilidades propostas nas DCN.

Descritores: Educação Superior. Estudantes de Odontologia. Relações ComunidadeInstituição.

\section{INTRODUÇÃO}

Historicamente, o modelo de formação do profissional de saúde no Brasil foi marcado por ignorar estratégias pedagógicas fundamentadas no ensino problematizado e na construção do saber coletivo, devido à utilização de metodologias tradicionais de 
ensino que priorizavam a superespecialização e a sofisticação dos procedimentos ${ }^{1}$. Não diferente disto, a formação na Odontologia visava uma atenção elitizada, desassociada da noção de bem-estar e de qualidade de vida, sendo acessível apenas a uma pequena parcela da população ${ }^{2}$.

Com o intuito de preencher algumas lacunas na formação profissional de acadêmicos, as Diretrizes Curriculares Nacionais $(\mathrm{DCN})^{3}$, vigentes desde 2002 para o curso de Odontologia, buscam formar um profissional com capacidade generalista, porém com uma visão mais humana, crítica e reflexiva, que tenha como base um rigor técnico e científico. Além disso, devem ser capacitados a atuar na saúde bucal da população em diversos cenários, pautado em princípios éticos e legais, compreendendo a realidade social, cultural e econômica do seu meio, dirigindo sua atuação para a transformação da realidade em benefício do ser humano e da sociedade ${ }^{3-5}$.

Para a formação de profissionais com este perfil, as universidades, em seu projeto político pedagógico, traçaram dentre os objetivos a efetivação da prática odontológica como eixo articulador dos conhecimentos a serem construídos ao longo do curso, por meio de projetos interdisciplinares e de contextualizações que possibilitem abranger as três dimensões da formação universitária: a pesquisa, o ensino e a extensão ${ }^{3}$.

Dentro desta tridimensionalidade, a extensão universitária, através de sua configuração de processo educativo cultural, científico e educacional, é a que melhor permite a articulação entre o ensino e a pesquisa de forma indissociável, além de viabilizar a relação transformadora entre universidade e sociedade $^{6}$. Ademais, configura-se como um excelente cenário de metodologias ativas de aprendizado, pois possibilita formar profissionais capazes de aplicar na prática os conhecimentos adquiridos em sala de aula, permitindo uma reflexão sobre seu papel nesses cenários e, por consequência, buscar sempre soluções para mudar a realidade de sujeitos e coletividades $^{7,8}$.

Dentro desta perspectiva, o presente estudo buscou analisar qualitativamente a percepção de acadêmicos de Odontologia acerca de um programa extensionista, sob a ótica da aproximação com as Diretrizes Curriculares Nacionais do referido curso.

\section{METODOLOGIA \\ Os sujeitos}

Constituíram-se sujeitos desta pesquisa, qualitativa descritiva, acadêmicos de Odontologia de uma universidade pública do estado de São Paulo, com idade média de 20 anos, participantes do "Programa de Atenção Odontológica à Gestante”, no ano de 2014.

Foi incluída na amostra a totalidade de acadêmicos com índice igual ou superior a $75 \%$ de participação nas atividades anuais desenvolvidas pelo programa $(n=5)$.

\section{O Programa}

O "Programa de Atenção Odontológica à Gestante" atua junto a gestantes matriculadas no programa de pré-natal, do serviço público de saúde de um município do noroeste paulista, desenvolvendo ações de avaliação do risco de desenvolvimento de doenças bucais; intervenções clínicas; e reuniões didáticopedagógicas.

Uma das metodologias utilizadas pelo programa baseia-se na pedagogia da problematização, abordagem na qual uma situação específica atua como estímulo para a aprendizagem ativa ${ }^{9,10}$. Os acadêmicos participantes do programa identificam a realidade e as possíveis dificuldades na área de 
campo e reúnem-se em rodas de discussão para planejar ações e, posteriormente, voltam a campo e colocam em prática a estratégia de eleição.

Paralelamente a essas atividades são desenvolvidas outras metodologias ativas de aprendizado junto aos acadêmicos envolvidos, como oficinas, palestras de capacitação e pesquisas científicas, objetivando instrumentalizá-los sobre formação em saúde maternoinfantil, com base em evidência científica ${ }^{12}$.

\section{Meios para obtenção das informações}

Os elementos de interesse para o estudo foram apreendidos por meio da técnica de entrevista em grupo focal. Para o desenvolvimento da técnica um pesquisador previamente treinado conduziu a entrevista orientando-se por um roteiro de dez tópicos, elaborado no sentido de nortear a conversa e apreender o ponto de vista dos sujeitos entrevistados sobre a temática 'a contribuição do Programa de Atenção à Gestante na vida acadêmica', abordando aspectos como o desenvolvimento acadêmico e pessoal; as sensações de trabalhar com gestantes e diferentes profissionais da saúde; a oportunidade em trabalhar em equipe; as relações entre a pesquisa, ensino e extensão.

Durante toda a entrevista, o pesquisador permitiu o livre discurso e ao mesmo tempo o delineamento da conversa, buscando manter a entrevista sintonizada com os objetivos da pesquisa. A entrevista foi gravada, teve duração de cinquenta minutos e para seu encerramento utilizou-se o critério de saturação ${ }^{12}$.

\section{Análise das informações}

Os dados coletados foram primeiramente transcritos na íntegra para depois serem tratados de acordo com a técnica de análise do Discurso do Sujeito Coletivo
(DSC), proposta por Lefèvre e Lefèvre $(2003)^{13}$.

$\mathrm{Na}$ primeira etapa foram identificadas as expressões-chave de conteúdo similar, e as ideias centrais, seguindo os critérios de exaustividade, representatividade, homogeneidade e pertinência. Posteriormente as expressões orais, de vários discursos individuais, com ideias similares foram agrupadas em um único discurso-síntese.

Este trabalho foi aprovado pelo Comitê de Ética em Pesquisa da Faculdade de Odontologia de Araçatuba - FOA/UNESP (Parecer $\mathrm{n}^{\circ}$ 1.235.099), respeitando os ditames da resolução 466/12 do Conselho Nacional de Saúde.

\section{RESULTADOS E DISCUSSÃO}

Foram gerados na análise seis discursos do sujeito coletivo (DSC), cada qual formado por uma ideia central e distribuído entre os três temas resultantes da análise, a saber: Desenvolvimento Científico; Desenvolvimento Técnico e; Desenvolvimento das Relações Humanas. Os achados são apresentados e discutidos em seguida com aporte teórico.

\section{Tema 1 - DESENVOLVIMENTO CIENTÍ-} FICO

IDEIA CENTRAL - Desbravando as envergaduras do campo científico

DSC - Para o desenvolvimento acadêmico pra mim foi importante, porque eu comecei a adquirir além de experiência, conhecimento [...] conhecimento de saúde pública, conhecimento na prática. Ah e foi importante também pro currículo né! Nosso Lattes fica com as horas lá, apresentação de trabalho, aqui a gente tem essa possibilidade de fazer muitos trabalhos, comecei escrever artigos... Ah 
eu tô aprendendo muita coisa [...] então pra quem quer, por exemplo, mestrado isso é ótimo.

No discurso dos acadêmicos fica evidente a possibilidade angariada pela extensão universitária em adquirir conhecimento dentro do contexto vivenciado e exercer na prática os conhecimentos obtidos em aula, oportunizando a reflexão, sentido e aplicabilidade dos conteúdos teóricos apreendidos, aprofundando seu entendimento acerca da saúde coletiva na sua formação. A contextualização do aprendizado com a realidade estimula o olhar comparativo, crítico e enaltece a formação multifocal, congregando distintas competências e habilidades necessárias aos profissionais da saúde ${ }^{14}$.

Além disso, é evidente o fortalecimento das habilidades propostas no artigo $5^{\circ}$ das DCN como "conhecer métodos e técnicas de investigação e elaboração de trabalhos acadêmicos e científicos; analisar e interpretar os resultados de relevantes pesquisas; $e$ participar de investigações científicas sobre doenças e saúde bucal e estar preparado para aplicar os resultados de pesquisas para os cuidados de saúde"3.

Os achados do presente estudo endossam também o pressuposto previsto na legislação brasileira ${ }^{15}$ para as universidades, ao evidenciar, dentro do programa de extensão avaliado, o princípio da indissociabilidade entre o tripé ensino, pesquisa e extensão. De acordo com Moita e Andrade (2009) ${ }^{16}$ a indissociabilidade é norteadora da qualidade da produção universitária, visto que subsidia a formação, através da tridimensionalidade, do profissional autônomo, competente e ético e mais preparado para a vida profissional.

No entanto, apesar de sua importância, no Brasil, a extensão ainda não consegue atingir o caráter de igualdade e indispensabilidade frente à formação profissional, quando comparado ao ensino e a pesquisa ${ }^{14}$.

Deste modo, fazer da extensão um campo de que vai além da formação, que se preocupa com os problemas da sociedade contemporânea, que envolva também o campo do saber científico, é fundamental para impedir os reducionismos que se verificam na prática universitária, tais como a ênfase apenas na produção do novo saber, ou meramente na transmissão de conhecimentos na formação profissional ${ }^{16}$; e ainda para fomentar mudanças significativas nos processos de ensino e aprendizagem e colaborar efetivamente para a formação profissional e cidadã dos sujeitos envolvidos.

\section{Tema 2 - DESENVOLVIMENTO TÉC- NICO}

IDEIA CENTRAL - Explorando a prática integral à saúde com gestantes

DSC - A gente não tem na graduação nada voltado para gestante, e têm $n$ estágios que é assim, uma complementação do que a gente vê na graduação. Então é diferente, aqui a gente adquire conhecimento de tratamento, né, técnico, atendimento, de como tratar a gestante, na prática. [...] Antes eu nem sabia da importância de educar uma gestante, mostrar pra ela a importância de ter uma boa higienização, de procurar sempre um dentista durante a gestação, eu mesma nem sabia. [...] É um diferencial que a gente tem aqui, é um aprendizado a mais que a gente vai ter aqui e levar para clínica.

Poucas universidades brasileiras oferecem em seu currículo a formação técnicocientífica e a prestação de serviço para atender gestantes durante o curso de graduação ${ }^{4}$. Este despreparo pode levar a insegurança de 
profissionais, tanto na execução de procedimentos quanto no uso de medicações, visto que o período gestacional é repleto de mitos e tabus em relação ao tratamento odontológico ${ }^{4}$.

O programa de extensão veio como meio de suprir essa lacuna na formação dos futuros cirurgiões-dentistas e contribuir para que eles ampliem as suas formas alternativas de atuação e execução do trabalho e aquisição de conhecimento; criem o poder de responsabilização; e aprimorem suas habilidades e atitudes adquiridas nas diferentes disciplinas.

Além disso, pode-se observar no discurso a visão de integralidade na atenção, sugerindo que a extensão estimulou o acadêmico a valorizar os processos educativo/preventivos, que representam a chave do sucesso almejado pela atenção primária à saúde ${ }^{17}$. Contemplando na formação do indivíduo a habilidade de atuar em todos os níveis de atenção à saúde, entendidos como conjunto articulado e contínuo das ações e serviços de promoção, manutenção, prevenção, proteção e recuperação da saúde ${ }^{3}$.

Ainda, ajudou a desmistificar a figura do cirurgião dentista como um profissional que realiza apenas procedimentos clínicos na cavidade bucal e trabalha isolado em ambiente de consultório odontológico.

\section{IDEIA CENTRAL - Aprimorando a desen-} voltura, postura e sustentação oral

DSC - Eu tinha muita vergonha, tinha um grande problema de conversar principalmente com pessoas que eu não conhecia, como os nossos pacientes por exemplo. Então para falar com gestantes eu acabei aprendendo a me soltar um pouco mais. Além disso, quando a gente ministrou as capacitações, tivemos que dominar um assunto que era totalmente desconhecido... Isso tudo ajudou bastante, hoje eu tenho segurança quando eu vou apresentar palestras, alguma coisa, eu consigo me soltar, eu consigo me interagir muito com um público.

Segundo as DCN (2002) $)^{3}$, atenção à saúde, tomada de decisões, comunicação, liderança, administração e gerenciamento são habilidades necessárias para a formação do cirurgião-dentista, para que o mesmo saiba atuar com cidadania e ética, nos níveis individual e coletivo.

Para Santos $(2012)^{18}$ a comunicação envolve falar com clareza e objetividade, dominar um assunto, saber organizar ideias e ter postura corporal, e devem ser desenvolvidas e aprimoradas ao longo da formação profissional.

Como observado, a atividade de extensão causou uma mudança nos acadêmicos participantes, propiciando a eles maior segurança, autonomia para se relacionar e comunicar de maneira efetiva frente a diferentes conjunturas. Vivenciar situações externas ao ambiente da universidade faz com que o acadêmico supere seus medos e limites em diversas situações, como a comunicação com as pessoas ${ }^{19}$.

Além disso, Santos et al. $(2013)^{20}$ afirmam que os acadêmicos de Odontologia participantes de atividades de extensionistas têm melhor compreensão dos casos clínicos, conseguindo tomar decisões e transmitir as possibilidades de tratamento para paciente com maior facilidade, assim como resolver os problemas na sua área de atuação.

Todas estas habilidades e competências também se estendem ao trabalho isolado no consultório, onde o profissional necessitará delas para atender seus pacientes com mais responsabilidade e dignidade, tendo em conta que o seu compromisso com o paciente não se encerra com o ato técnico. 
IDEIA CENTRAL - Interação com o serviço público de saúde

DSC - O contato que a gente tem na UBS é maravilhoso, porque eu não conhecia como que era o sistema público de saúde... eu nunca tinha o costume de ir no SUS, essas coisas, e você vê que é uma realidade totalmente diferente... tem vários profissionais juntos, a enfermeira chefe organiza, marca tudo. Quer saber, eu não sabia mesmo como funcionava, $e$ agora aqui a gente aprende tudo isso, isso daí é muito legal... importante!

Algumas instituições de ensino superior desde muito tempo preocupam-se em proporcionar ao acadêmico a atuação nos mais diversos cenários de aprendizagem e conviver com distintas realidades, através da atuação nos serviços extramurais e nas extensões universitárias ${ }^{21}$.

Essa preocupação vem aumentando nos últimos anos, em especial no reconhecimento da área de atuação dos profisssionais da saúde inseridos nos serviços públicos. Até mesmo o governo federal passou a investir de iniciativas como o Programa de Educação pelo Trabalho e Saúde (PET-Saúde) e Programa Nacional de Reorientação da Formação Profissional em Saúde e (PRO-Saúde) com vistas a formar profissionais mais qualificados para atender as necessidades de seu sistema de saúde ${ }^{22}$.

De acordo com Santos et al., $(2013)^{20}$ a interação ativa do aluno com a população e os profissionais de saúde é crucial para o processo de formação, pois induz o acadêmico a formular estratégias para trabalhar com problemas reais, assumindo responsabilidades crescentes. Além disso, o empodera para ter noções melhores de gerenciamento e administração, tanto da força de trabalho, dos recursos físicos e materiais e de informação, facilitando as aptidões para ser empreendedor, gestor, empregador ou líder na equipe de saúde. Ainda, dá a oportunidade de compreender as políticas de saúde bucal, o papel do cirurgião-dentista e de todos os membros da equipe ${ }^{20}$. Estes achados coadunam com o exposto pelos acadêmicos entrevistados.

Deste modo, quem tem oportunidade de vivenciar ações fora dos muros da universidade, desfrutará de formação mais qualificada, diferenciada, com maior senso crítico, interativo, comunicativo, proativo e preparado para atuar de forma adequada dentro da dinâmica e das exigências do SUS.

Tema 3 - DESENVOLVIMENTO DAS RELAÇÕES HUMANAS

IDEIA CENTRAL - Prerrogativas do trabalho em equipe

DSC - Pra mim foi muito gostoso esse contato com professores, pósgraduandos e com graduandos também. Os mais velhos (no Programa) passaram o conhecimento pra nós, de maneira muito informal. A pós também ajudou a gente bastante, nas questões das palestras, sempre complementou tudo o que a gente fez, ajudou a gente aprender ainda mais. Então, a gente sempre tá convivendo, tendo que trabalhar em equipe. $O$ retorno em trabalhar em equipe é melhor, além de não sobrecarregar e distribuir funções. Eu tenho certeza que a gente vai levar muito desse conhecimento pra nossa vida profissional aí fora.

Segundo Santos et al. $(2014)^{19}$, o trabalho em equipe é fundamental e implica na busca de comunicação constante, afim de se obter uma troca de experiências e conhecimentos entre os diferentes sujeitos, visando a construção de consensos e acordos, mesmo que provisórios. Habilitar o profissional a trabalhar em equipe multidisciplinar é uma 
das finalidades da formação do cirurgiãodentista $^{3}$.

A fala dos sujeitos deixa claro que o programa de extensão foi capaz de criar um ambiente propício para oferecer treino sistemático para os sujeitos envolvidos de como trabalhar cooperativamente em equipe, ensinando-os a desenvolver comportamentos compatíveis para o êxito do grupo. $\mathrm{O}$ achado corrobora com o estudo de Fadel et al. $(2013)^{23}$ que expõe que a extensão universitária é capaz de promover maior convivência, troca de conhecimentos e respeito ao saber alheio, além de facilitar o aprendizado do trabalho em equipe.

Deste modo, pode-se considerar a extensão universitária como uma estratégia exitosa para formar profissionais com perfil para o trabalho em equipe. Perfil este, bastante valorizado no mercado de trabalho atual ${ }^{24}$. Quem apresenta este perfil tem em mãos o diferencial para lidar com as formas de interagir, com as diferenças, uma capacidade ímpar de comunicação, com uma postura proativa, de parceira e de cumplicidade e com uma visão integrativa, capaz de uma atuação mais efetiva e pertinente com seu campo de atuação ${ }^{24,25}$.

Essa valorização do trabalho em equipe é vislumbrada especialmente no serviço público de saúde, onde o trabalho de forma multiprofissional é fator decisivo na busca da atenção integral, pois favorece maior integração entre os profissionais de saúde e as ações que desenvolvem, facilitando o planejamento e a organização do trabalho ${ }^{24}$.

IDEIA CENTRAL - Aproximação com a realidade da população

DSC - O conhecimento da realidade, esse contato com a comunidade, com a população, lá onde eles moram, onde eles vivem, me fez enxergar o quanto que a população precisa da gente, que pessoas que não têm instrução nenhuma faz coisas que, às vezes, é absurda pra gente... Às vezes, eu explicava alguma coisa, eu achava um absurdo eles não entenderem. E eu vendo a realidade deles, eu entendi porque que eles não entendem. Por que que é mais difícil? Porque eles não têm esse conhecimento mesmo. Isso me fez atender os pacientes na clínica, de um modo diferente, mais humanizado.

As DCN reforçam a necessidade de inserir acadêmicos em cenários reais de práticas dos serviços de saúde como estratégia para a diversificação de ambientes de atuação, para que os mesmos consigam visualizar e exercer seu papel como agente social modificador, além de aflorar a criatividade para soluções de problemas.

A atuação na realidade é um campo ideal para aplicabilidade de metodologias ativas de aprendizagem. No programa de extensão, a utilização da metodologia de aprendizagem baseada em problemas dá ao aluno a possibilidade de atuar diretamente em todas as etapas do processo da problematização que vão desde o conhecimento e diagnóstico do problema até a execução das ações para a sua solução.

O uso da problematização como estratégia de ensino permite tornar os estudantes mais ativos, pensadores críticos, capazes de perceber seu desenvolvimento, com competência profissional, além de possibilitar a continuidade do aprendizado ao longo da vida, de maneira interdisciplinar e integral, conseguindo atingir todos os níveis de atenção à saúde ${ }^{8}$.

A importância que o conhecimento de distintas realidades e a prática baseada em problemas causou nos estudantes pode ser 
observada em suas falas. A visualização e compreensão da população, por eles assistidas, em seu meio social e cultural, tornou-os mais sensibilizados e comprometidos com a saúde do ser humano, sabendo, assim, respeitá-los e valorizá-los dentro de seu contexto. Além disso, impulsionou-os no desenvolvimento de competências para transformação da realidade em benefício do ser humano e da sociedade.

Deste modo, quando o acadêmico reconhece os ganhos e a aprendizagem que participar desse tipo de atividade lhe proporcionou, é sinal de que sua formação como pessoa e cidadão saiu fortalecida ${ }^{19}$.

Conforme o exposto, a participação ativa em um programa de extensão o qual tem fortalecida a indissociabilidade entre ensinopesquisa-extensão, atua dentro de diferentes cenários, possui uma forte interação com o serviço de saúde e a comunidade, e trabalha com metodologias ativas de aprendizagem, configura-se como uma excelente oportunidade de formação profissional contextualizada com as habilidades propostas nas DCN para o curso de Odontologia.

Contudo, pela não obrigatoriedade regida nas DCN para implementação de estágios com estas características, algumas instituições acabam, por diversas vezes, limitando-se em atividades intramurais, complementando a carga horária de estágios obrigatórios com estágios clínicos ou laboratoriais.

Assim sendo, sugere-se o repensar das DCN vigentes para os cursos de Odontologia, com inclusão, nos estágios supervisionados, de atividades extramurais que contemplem as diversas vertentes, cenários e metodologias ativas de aprendizado.

\section{CONCLUSÃO}

O programa extensionista, na ótica dos acadêmicos entrevistados, revelou ser um importante agente para o processo individual e coletivo de formação acadêmica, que não se limita ao tradicional, uma vez que, contribui para uma formação em saúde mais humanista, generalista e preparado para atuar frente à situação de saúde do país, como preconizam as diretrizes curriculares.

\section{ABSTRACT \\ University extension in the view of academics: the enabler of National Curricular Guidelines} This study aimed to analyze the perception of dental students about an extension program, from the perspective of rapprochement with the National Curricular Guidelines (DCN) of that course. It is a qualitative study, developed with academics $(n=5)$ participating in an outreach program geared to prenatal. Data were collected through focus groups and analyzed by the Collective Subject Discourse technique. It was possible to observe that the impact exerted by academic experience in extension activities enhances the scientific, technical and human relations. In the field of scientific development, the gain was in receiving stimuli to develop their skills to seek new knowledge and fix the theoretical content in practical experience. Concerning in the technical development, there was improvement of the technical-scientific and practical knowledge to humanized attention to pregnant women, as well as development motor and cognitive skills (to understand the functioning of the public health system), and communication and academic expression. In the field of development of human relations, the fruitful relationship established between academic, university and society allowed to increase the skills of teamwork and a sense of citizenship. It concludes that an outreach program sets up an excellent professional training opportunity, more contextualized with the proposed skills in DCN.

Descriptors: Education, Higher. Dental Students. Community-Institutional Relations. 


\section{REFERÊNCIAS}

1. Moraes BA, Costa NMSC. Análise documental de currículos na área da saúde no Brasil. CIAIQ 2015;1:224-8.

2. Fonsêca GS, Rodrigues AÁAO. O Programa de Educação pelo Trabalho para Saúde (PET-Saúde) como indutor de inovações pedagógicas: a experiência do curso de Odontologia da Universidade Estadual de Feira de Santana, Bahia. Rev ABENO 2011;11(2):19-26.

3. Brasil. Ministério da Educação. Conselho Nacional da Educação. Câmara da Educação Superior. Resolução CNE-CES n. 3. Instituiu as Diretrizes Curriculares Nacionais do Curso de Graduação em Odontologia. Brasília, 2002. Disponível em: http://portal.mec.gov.br/cne/arquivos /pdf/CES032002.pdf

4. Moimaz SAS, Saliba NA, Garbin CAS, Zina LG, Furtado JF, Amorim JA. Serviço Extra-Mural Odontológico: Impacto na formação profissional. Pesq Bras Odontoped Clin Integr 2004;4(1):53-7.

5. Bispo NTB, Cecchin D, Zanette F, Grazzi otin-Soares R. Aquisição de conhecimento de estudantes de odontologia da UPF durante a graduação: avaliação sob parâmetros do Enade. RFO 2012;17(3):273-9.

6. Brasil. Política Nacional de Extensão Universitária. Fórum de Pró-Reitores de Extensão das Universidades Públicas Brasileiras. Manaus, 2012. Disponível em: http://www.renex.org.br/documentos/2012 -07-13-Politica-Nacional-de-Extensao.pdf

7. Pedrosa IL, Lira GA, Oliveira B, Silva MSML, Santos MB, Silva EA, Freire DMC. Uso de metodologias ativas na formação técnica do agente comunitário de saúde. Trab Educ Saúde 2011; 9(2):31932.
8. Silva RHA, Miguel SS, Teixeira LS. Problematização como método ativo de ensino-aprendizagem: estudantes de farmácia em cenários de prática. Trab Educ Saúde 2011;9(1):77-93.

9. Bassir SH, Sadr-Eshkevari P, Amirikhorheh S, Karimbux NY. Problem-based learning in dental education: a systematic review of the literature. J Dent Educ 2014;78(1):98-109.

10. Gharechahi M, Zarei M, Javidi M. Evaluation of students' opinion about seminars conducted with problem based learning method in endodontics department of Mashhad Dental School. Future Med Educ J 2014;4(1):22-6.

11. Moimaz SAS, Gomes AMP, Bordin D, Garbin CAS, Saliba NA. Extensão universitária como ferramenta geradora de ensino-aprendizagem e produtora de pesquisa. Rev Conex UEPG 2015; 11(2):140-9.

12. Veiga L, Gondim SMG. A utilização de métodos qualitativos na Ciência Política e no Marketing Político. Opin Publica 2001; 7(1):1-15.

13. Lefèvre F, Lefèvre AMC. O Discurso do Sujeito Coletivo: um novo enfoque em pesquisa qualitativa (Desdobramentos). Caxias do Sul: EDUCS, 2003.

14. Cardoso AC, Corralo DJ, Krahl M, Alves LP. O estimulo à prática da interdisciplinaridade e do multiprofissionalismo: a Extensão Universitária como uma estratégia para a educação interpor fissional. Rev ABENO 2015;15(2):12-9.

15. Brasil. Constituição da República Federativa do Brasil de 1988. Diário Oficial da União. Brasília, 1988. Disponível em: http://www.planalto.gov. br/ccivil_03/Constituicao/DOUconstituica o88.pdf 
16. Moita FMGSC, Andrade FCB. Ensinopesquisa-extensão: um exercício de indissociabilidade na pós-graduação. Rev Bras Educ 2009;14(41):269-80.

17. Moura LFAD, Piauilino RJB, Araújo ÍF, Moura MS, Lima CCB, Evangelista LM, Lima MDM. Impacto de um projeto de extensão universitária na formação profissional de egressos de uma universidade pública. Rev Odontol UNESP 2012;41(6):348-52.

18. Santos AB. Extensão universitária como viabilizadora de políticas públicas: a visão de acadêmicos da EDESC. 2012. 98 f. Dissertação (Mestrado Profissional em Gestão de Políticas Públicas) Universidade do Vale do Itajaí, Itajaí, Santa Catarina. 2012.

19. Santos SPF, Ranghetti DS, Gascho JA. Inclusão digital com idosos: projeto de extensão para educação integral. Cataventos 2014;6(1):156-75.

20. Santos KT, Ferreira L, Batista RJ, Bitencourt CTF, Araújo RP, Carvalho RB. Percepção discente sobre a influência de estágio extramuro na formação acadêmica odontológica. Rev Odontol UNESP 2013;42(6):420-5.
21. Saliba NA, Saliba O, Moimaz SAS, Garbin CAS, Arcieri RM, Lolli LF. Integração ensino-serviço e impacto social em cinquenta anos de história da saúde pública na Faculdade de Odontologia da Universidade Estadual Paulista Júlio de Mesquita Filho. RGO 2009;57(4):459-65.

22. Brasil. Ministério da Saúde Pró Saúde. Disponível em: http://www.prosaude.org/. Acesso em 20 set. 2015.

23. Fadel CB, Bordin D, Kuhn E, Martins LD. O impacto da extensão universitária sobre a formação acadêmica em Odontologia. Interface 2013;17(47):937-46.

24. Otenio CCM, Nakama L, Lefèvre AMC, Lefèvre F. Trabalho Multiprofissional: representações em um serviço público de saúde municipal. Saúde Soc 2008; 17(4):135-50.

25. Cardoso CL. Relações interpessoais na equipe do Programa Saúde da Família. Revista APS 2004;7(1):47-50.

\section{Correspondência para:}

Adrielle Mendes de Paula Gomes e-mail: adrielle_mendes@ hotmail.com Rua José Bonifácio, 1193 - Seção de PósGraduação 16015-050, Vila Mendonça, Araçatuba - SP 